\title{
Premorbid Negative Symptoms in First-Episode Psychosis
}

\author{
Manuel J. Cuesta* \\ Victor Peralta* \\ Patxi Gil ${ }^{\star \star}$ \\ Maria Artamendi** \\ * Psychiatric Unit of Virgen del Camino \\ Hospital, Pamplona \\ ** Vasque Health System - Osakidetza, \\ Vitoria
}

SPAIN

\begin{abstract}
Background and Objectives: Negative symptoms emerge in many patients with psychotic disorders long before the onset of the acute illness. These symptoms are often impossible to differentiate from certain Cluster A personality traits.

Methods: The current study examines the extent to which premorbid negative symptoms are contributing factors to the development of primary and secondary negative symptomatology. Participants were 84 neuroleptic-naïve patients experiencing the occurrence of their first acute psychotic episode. Symptoms of psychopathology were assessed at two points: at admission and after remission of the acute episode. The Spanish version of the PANSS scale was administered. Premorbid personality assessment was considered as a proxy measure to evaluate each participant's negative symptomatology prior to the onset of the illness. Potential causes of secondary negative symptomatology, such as depression and extrapyramidal symptoms, were also examined.

Results: 'Non-respondent' or 'residual' negative symptoms at discharge were significantly predicted by primary negative symptoms. To a lesser extent, disorganization and depressive symptoms at discharge and the Schizoid dimension of premorbid personality predicted residual negative symptoms.

Conclusions: The severity of negative symptoms at the onset of the psychotic episode varied across patients. After controlling for 'respondent' and 'non-respondent' primary negative symptoms and other potential causes of negative symptoms, premorbid negative symptoms had a slight, but significant predictive relationship with residual negative symptoms.
\end{abstract}




\section{Introduction}

Schizophrenia and other psychotic disorders are heterogeneous conditions that are best understood as complex ${ }^{1}$, lifelong disorders ${ }^{2}$. Approaches to the study of the symptomatology of schizophrenia have not rendered a definitive profile of the characteristic manifestations of this disorder. Polythetic definitions, such as those included in the Diagnostic Statistical Manual and ICD-10 classification systems, continue to govern the process of establishing a diagnosis of schizophrenia.

There has not been one clinical entity, syndrome or dimension within the realm of psychosis that has been externally validated. However, negative symptoms are usually considered closer in relation to neurobiological substrates than other symptomatologic domains. Nevertheless, negative symptoms are neither necessary nor sufficient criterion to diagnose a schizophrenic disorder. Negative symptoms are not uncommon in other mental (e.g., Depressive episodes) and brain disorders (e.g., Alzheimer's Disease). The presence of negative symptoms has been demonstrated in relatives of many schizophrenia patients ${ }^{3}$. It is often assumed that negative symptoms are abnormal conditions, but normative studies have not been conducted. In addition, negative symptoms are very close to the specific criteria for a number of personality disorders (i.e., Schizoid, Avoidant and Schizotypal personality disorders $)^{4,5}$.

The latter statement lends support to the assertion that not all patients begin psychotic episodes with the same level of intensity of negative symptoms. To label these negative symptoms, Tandon et al. ${ }^{6}$ coined the term premorbid negative symptoms. In comparison, primary negative symptoms, on grounds of hypothesized causality, are best understood as the negative symptoms that are displayed at the onset of the acute episode of the illness, after ruling out the contribution of potential secondary sources of negative symptoms. Therefore, the negative symptomatology of the first acute episode is characterized by the addition of the primary negative symptoms to the premorbid negative symptoms plus other negative symptoms caused by other conditions (e.g., depressive anhedonia, social isolation or neuroleptic akinesia). The primary negative symptoms are considered illness-related symptoms that may respond, at least in part, to antipsychotic treatment ${ }^{7-9}$. Nonrespondent or residual primary negative symptoms were predicted mainly by the primary negative symptoms, after controlling for other potential contributors, such as depression, neuroleptic-induced extrapyramidal side effects, social isolation and other factors $^{10-13}$. However, studies have not been conducted to address the extent to which premorbid negative symptoms determine further negative symptomatology over the course of the illness.

Different approaches can be taken to delineate the influence of premorbid negative symptoms on primary and secondary negative symptoms, although follow-up studies are the most appropriate method to employ. However, the presence of negative symptoms can be traced back using indirect approaches, such as the assessment of premorbid personality. Premorbid personality traits are currently reliably quantified through modern semi-structured interviews. These traits are nearly identical to certain negative symptomatologic domains; therefore the assessment of premorbid personality traits might be used as a proxy measure of an individual's premorbid negative symptomatology. 
Previous studies from our group and others have demonstrated a close relationship between premorbid personality dimensions and the symptomatology of acute episodes in samples of individuals with both chronic and recent-onset psychosis ${ }^{14-17}$. A common limitation in the literature on premorbid personality, including one of our previous studies ${ }^{18}$ was that negative symptomatology was examined during the remission of the acute episode. Thus, only residual negative symptoms were addressed without controlling for primary symptomatology. In an effort to overcome this shortcoming and to develop a better understanding of the origin and nature of negative symptoms in early psychosis, it is essential to not only control for the effects of secondary negative symptoms, but also for the contribution of primary negative symptoms and other potential causes of negative symptomatology. We hypothesized that certain domains of the premorbid personality are true expressions of premorbid negative symptoms.

\section{Methods}

\section{Participants}

Participants were 84 inpatients at Santiago Apóstol Hospital, an acute psychiatric unit in Vitoria, Spain. The patients were referred to the study because they had experienced a first-episode of acute psychosis. Acute psychotic episodes were defined by a score of 4 or greater on any of the following three items on the Positive and Negative Syndrome Scale (PANSS) ${ }^{19}$ : delusions, conceptual disorganization, or hallucinatory behavior. Diagnosis was based on the Structured Clinical Interview for DSM-IV $(\mathrm{SCID})^{20}$. Patients were assigned to the following DSM-IV diagnostic categories ${ }^{21}$ :
Schizophrenic Disorder $(n=28)$, Schizophreniform Disorder $(n=11)$, Brief Psychotic Disorder $(n=18)$, Delusional Disorder $(n=7)$, Manic Disorder with Psychotic Symptoms $(n=18)$ and Major Depressive Disorder with Psychotic Symptoms $(n=2)$.

Patients were between 18 and 65 years of age. Exclusion criteria included systemic or neurological illness, history of head trauma, and substance dependence. Ten patients were excluded because they had previously received anti-psychotic drugs. As a result, the final study sample consisted of 84 neuroleptic-naïve psychotic patients. An exhaustive investigation of previous drug treatments was conducted through information obtained from various sources (i.e., patient, relatives). Primary physician records were tracked when it was deemed necessary. All patients gave written informed consent before entering the study.

\section{Design and Assessments}

This study consisted of a reanalysis of data from a previous study ${ }^{22}$, which examined the relationships between premorbid personality dimensions and psychopathological dimensions at discharge. Three innovations were introduced in the present study in order to advance the understanding of the origin of negative symptoms in psychosis and to justify the present re-analysis. First, negative symptomatology at admission was included as a measure of primary negative symptoms in the statistical procedures, after accounting for by the remaining potential causes of negative symptoms at this initial assessment. Second, the premorbid personality dimensions were used as a proxy measure of premorbid negative symptoms. And third, only the neuroleptic-naïve subset of first episode psychotic patients was includ- 
ed in order to avoid interference in the assessment of premorbid personality. The design of this study enabled us to have three methods of the assessment of negative symptoms in order to answer our main research question: Were premorbid negative symptoms predictive of the intensity of residual negative symptomatology, after controlling for the influence of primary negative symptomatology?

Psychopathological symptoms were assessed with the Spanish adaptation of the PANSS. Participants were assessed on admission to the psychiatric unit (T1) and at discharge from hospital (T2). As reported by Marder et al. $^{23}$, we chose the five-dimensional model of the PANSS in order to extract the PANSS underlying dimensions. This model was comprised of the following dimensions: negative, psychotic, disorganization, excited and depressive. Neurolepticinduced extrapyramidal side effects were assessed at $\mathrm{T} 2$ by means of the total score on the Simpson-Angus Rating Scale ${ }^{24}$. The first author, MC, conducted all of the PANSS and Simpson-Angus Rating scale assessments.

The Personality Assessment Schedule $(\mathrm{PAS})^{25}$ was used to assess Premorbid Personality (PP). The PAS is a structured interview that evaluates 24 personality variables on a 9-point scale of intensity. An individual's score on each personality variable depends on the influence that the specific variable has had on their functioning over the course of their life. The PAS interview allows for the extraction of four clusters of personality traits: passive-dependent, obsessional, schizoid, and sociopathic. As we previously reported ${ }^{18}$ the PAS interview was partially modified to differentiate a schizotypic dimension.

In an attempt to avoid interference with current symptoms, we chose the patient's mother or father as the main informant. In order to collect measures of enduring personality characteristics, the questions that informants were asked about a participant's personality placed special emphasis on the premorbid period. As recommended by other researchers ${ }^{26}$, the premorbid period was defined as one year before the appearance of any prodromic manifestation or any noticeable clinical symptom. PG, who was blind to the psychopathological status of patients, administered the PAS interview.

\section{Statistical Analysis}

Psychopathological at both assessment points and personality scores were examined by means of the Kolmogorov-Smirnoff test. This statistical procedure was used to transform non-normally distributed scores to logarithm scaling in order to achieve normality. The first step of the statistical analysis used Spearman rank coefficient correlations to examine the associations between negative symptomatology at $\mathrm{T} 2$, later referred to in the text as 'residual' or 'nonrespondent' negative symptomatology, and its potential contributing factors. The latter included premorbid personality dimensions, symptomatological dimensions at admission, Simpson-Angus total score and nonnegative symptomatological dimensions at $\mathrm{T} 2$.

Stepwise linear regression analysis was then employed to determine the particular contributions of the variables that were found to be significantly associated with residual negative symptoms in the former correlational analysis. The coefficient of determination $\left(R^{2}\right)$, added at each step and multiplied by 100 , was used to designate the proportion of variance in residual negative symptoms (dependent variable) accounted 
for by its predictors. Measurement error that was not explained by the secondary sources of negative symptoms might also contribute to variability in the negative symptoms.

\section{Results}

The demographic and clinical variables of the patients are presented in Table I. As expected, there were significant differences between admission and discharge scores on the five psychopathological dimensions of the PANSS scale.

Associations between residual negative symptomatology and premorbid personality dimensions, psychopathological dimensions at T1 and T2, and total score of the Simpson-Angus scale are shown in Table II. Significant correlation coefficients were found between residual negative symptomatology and the following scores: premorbid personality dimensions of schizoid, passive-dependent and schizotypic, negative and disorganization symptoms at $\mathrm{T} 1$, disorganization and depressive symptoms at T2 and the total score of the Simpson-Angus scale at T2. The variables that had significant correlation coefficients with residual negative symptomatology were entered further in the regression analysis.

Forty-two percent of the variance in residual negative symptoms was accounted for by primary negative symptoms. At steps 2 and 3, disorganization symptoms at T2 (Adjusted $R^{2}=.13$ ) and depressive symptoms at T2 (Adjusted $R^{2}=.05$ ) were entered into the analysis. And finally, the schizoid premorbid personality dimension score predicted five percent of the variance in residual negative symptoms (Adjusted $R^{2}=.05$ ).

\section{Discussion}

Residual or 'non-respondent' negative symptoms were strongly predicted by primary negative symptoms. Primary negative symptoms are hypothetically related to the illness process. Other symptom domains, such as disorganization and depressive symptoms at discharge (T2), were also moderately, but significantly, associated with residual negative symptoms. These results were in accordance with previous results that we obtained with the data set from a different sample ${ }^{9}$. However, a novel finding in the current study was that not only did schizoid premorbid traits slightly, but significantly, predict the intensity of residual negative symptoms, but also that this effect persisted after controlling for the effect of the primary negative symptoms, other non-negative symptom domains at admission and discharge and the SimpsonAngus total score at discharge. To our knowledge, this study is the first to examine the contribution of premorbid negative symptoms to the differentiation between primary and residual negative symptomatology in psychosis.

Several explanations, referenced in works from classic literature to recent articles, have been proposed to account for the relationships between personality and the symptomatology of psychosis ${ }^{5,13,22}$. Briefly, premorbid personality has been considered to be a modulator, or pathoplastic agent, on schizophrenia symptoms, a comorbid disorder within Axis II ${ }^{21}$, a scar or residual symptomatology from earlier subthreshold psychiatric episodes, and a risk factor for developing psychosis. Collectively, these explanations provide for a more thorough understanding of these constructs. 
Table I

Demographic and clinical characteristics of the sample.

\begin{tabular}{lcc} 
& $\mathrm{N}$ & $\%$ \\
\hline Sex & 59 & 70.2 \\
Men & 25 & 29.8 \\
Women & & \\
\hline DSM-IV Diagnosis & 28 & 33.3 \\
$\quad$ Schizophrenic disorder & 11 & 13.1 \\
Schizophreniform disorder & 18 & 21.4 \\
Brief Psychotic disorder & 7 & 8.3 \\
Delusional disorder & 18 & 21.4 \\
Manic disorder with psychotic symptoms & 2 & 2.4 \\
Major depressive disorder with psychotic symptoms & & \\
\hline Previous treatment status & 69 & 17.9 \\
$\quad$ Never medicated & 15 & SD \\
Previously medicated but not with antipsychotic drugs & & 9.33 \\
& mean \\
\hline Age at hospitalization & 27.96 & 9.06 \\
Age at onset & 26.64 & \\
PANSS scales & & At
\end{tabular}

\begin{tabular}{lcrrr}
\hline & \multicolumn{2}{c}{ At admission } & \multicolumn{2}{c}{ At discharge } \\
\hline Positive syndrome & 26.38 & 6.56 & 12.67 & 4.64 \\
Negative syndrome & 21.61 & 8.98 & 16.75 & 6.71 \\
General Psychopathology & 43.06 & 10.24 & 27.88 & 6.40
\end{tabular}

PANSS dimensions

\begin{tabular}{lrrr}
\hline & At admission & At discharge & Change \\
\hline Negative & $2.85 \pm 1.45$ & $2.24 \pm 0.94$ & $-0.60 \pm 1.09^{1}$ \\
Psychotic & $3.70 \pm 0.79$ & $2.09 \pm 0.71$ & $-1.62 \pm 0.78^{2}$ \\
Disorganization & $2.65 \pm 0.85$ & $1.73 \pm 0.53$ & $-0.91 \pm 0.80^{3}$ \\
Excited & $2.84 \pm 1.35$ & $1.35 \pm 0.61$ & $-1.49 \pm 1.28^{4}$ \\
Depressive & $2.84 \pm 0.94$ & $1.82 \pm 0.65$ & $-1.01 \pm 0.97^{5}$ \\
Simpson-Angus scale & \multicolumn{3}{c}{ At discharge } \\
\hline
\end{tabular}

$1.47 \pm 2.34$

PANSS dimensions

\begin{tabular}{lll}
\hline Schizoid & 2.03 & 1.54 \\
Schizotypy & 1.27 & 1.36 \\
Sociopathic & 1.73 & 1.33 \\
Passive-Dependent & 1.89 & 1.20 \\
Anankastic & 1.68 & 1.05 \\
\hline
\end{tabular}

$n=84$; PANSS: The Positive and Negative Syndrome Scale; PAS: Personality Assessment Schedule; ${ }^{1}: \mathrm{z}=-.457$, $p=0.0000 ;{ }^{2}: \mathrm{z}=-.788, p=0.0000 ;{ }^{3}: \mathrm{z}=-.722, p=0.0000 ;{ }^{4}: \mathrm{z}=-.748, p=0.0000 ;{ }^{5}: \mathrm{z}=-.672, p=0.0000$. 
Table II

Correlation between secondary negative symptoms and potential predictors.

Residual Negative Symptoms (Negative symptoms at discharge)

r

DISCHARGE

Psychotic dimension at discharge

Disorganization dim at discharge

.19

Excited dimension at discharge

.42

Depressive dim at discharge

.07

Akinesia factor

.24

Drug factor

Simpson-Angus (Total Score)

\section{ADMISSION}

Negative dimension at admission

Psychotic dimension at admission

Disorganization dim. at admission

.33

.14

.30

Excited dimension at admission

.66

.04

.46

$-.10$

Depressive dim. at admission

\section{DIMENSIONS OF PREMORBID PERSONALITY}

Schizoid dimension $\quad .42$

Passive-dependent dimension $\quad .21$

Anancastic dimension $\quad-.01$

Sociopathic dimension $\quad .01$

Schitotypic dimension $\quad .23$

$r=$ Spearman correlation coefficients; $r=.21, p<0.05 ; r=.28, p<0.01 ; r=.31, p<0.005 ; r=.36, p<0.001$

Table III

Multiple regression analysis of significant predictors of negative symptom scores at admission and discharge and change between admission and discharge in 84 neuroleptic naïve patients with psychosis.

\begin{tabular}{lllllllll} 
& Model $F$ & df & $p$ & Beta & t & $p$ & Adj $R^{2}$ \\
\hline Predictors of negative symptom at discharge & & & & & & & & \\
$\quad$ controlled for negative symptom at admission & 56.85 & 1.78 & 0.0001 & & & & & \\
Negative dimension at admission & & & & .56 & 8.08 & 0.0001 & .42 \\
Disorganization dimension at discharge & & & & .26 & 3.62 & 0.0005 & .13 \\
$\quad \begin{array}{llll}\text { Depressive dimension at discharge } \\
\text { Schizoid dimension of personality }\end{array}$ & & & & .21 & 3.02 & 0.0034 & .05 \\
\hline
\end{tabular}

Our present design does not allow for a definitive testing of the goodness of fit of these models. However, studies of people at high-risk for psychosis that later develop first episode psychosis suggest that schizoid and other types of personality traits are risk factors for the development of schizophrenia ${ }^{27}$. As reported in schizotaxia ${ }^{28}$, predispositional states to schizophrenia, defined by the presence of negative symptoms, seem to be part of the genetic background to the illness. In our study, personality traits included within the schizoid dimension on the PAS were introspection, shyness and aloofness. These personality traits are considered very common in people at high-risk for psychosis. 
A comorbidity model is a simplistic view used to explain the coexistence of personality and symptomatologic domains. A comorbidity model would be valid in terms of description but it does not provide an underlying explanation. Another proposed model is the scar model, which maintains that personality traits would be secondary to a previous subthreshold or clinical episode. The latter seems to be an unlikely model for our study, since our sample consisted of patients experiencing their first psychotic episode.

Finally, the present results were in accordance with a pathoplastic contribution of premorbid personality traits to residual negative symptoms. An estimation of the contribution of premorbid personality traits was possible after we controlled for primary and secondary sources of negative symptomatology. This resulting estimation indicated that there was a small, but statistically significant contribution of premorbid schizoid traits to residual negative symptomatology in psychosis (about 5\% of the explained variance).

Our study also found that disorganization and depressive symptoms at discharge were moderate, but significantly associated with residual negative symptoms. However, this finding indicates more of an overlapping of psychopathological domains than of a true predictor effect, since depressive and disorganization dimensions at admission did not predict residual negative symptomatology. Moreover, medium to high correlations between these psychopathological dimensions are usual in clinical populations.

Several methodological limitations of the study warrant discussion. First, there is a wealth of evidence to suggest that a wide range of premorbid deviations precede the beginning of psychosis, therefore blurring the boundaries between premorbid and pro- dromal phenomena ${ }^{29}$. However, in accordance with suggestions made by other authors ${ }^{26}$ we chose the time frame of one year prior to the occurrence of noticeable prodromic or clinical symptoms as an arbitrary point for the establishment of the premorbid period. Second, as identified by other authors ${ }^{6}$ residual negative symptoms were defined as the negative symptoms that remained after the remission of the first psychotic episode (i.e. at discharge). Ideally, it would have been better to have a longer follow-up period, since many symptoms continue to improve beyond the resolution of the acute episode. Another potential shortcoming in this study was that extrapyramidal signs were not assessed at intake and could not be introduced into the regression analysis procedures. However, in a previous study conducted with a different sample, extrapyramidal symptomatology at intake did not significantly predict negative symptomatology at discharge ${ }^{9}$. Fourth, two potential causes of secondary negative symptomatology (e.g., chronicity and social isolation) were not included in our study. However, both chronicity and social isolation are sources of negative symptomatology, which usually emerge after successive episodes and not during the first episode of the illness. Fifth, the mixed diagnostic nature of sample, with predominance of schizophrenia disorders, would have biased the results regarding the negative symptomatology. However, by excluding affective patients $(n=20)$ the same significant associations, both in correlation and regression procedures, were found.

In summary, residual negative symptomatology was strongly associated with the primary negative or illness-related symptomatology ( $42 \%$ of explained variance). This strong, preceding relationship suggests a possible causal role for primary negative 
over 'non-respondent' negative symptomatology. Moreover, an additional 20\% of the explained variance in residual negative symptomatology resulted from the overlapping effect of disorganization dimension at T2 and depressive dimension at T2 (13\% and $5 \%$ of explained variance, respectively). Finally, the schizoid dimension of premorbid personality explained $5 \%$ of the variance in residual negative symptomatology. As a result, premorbid personality can be considered to be a true expression of the premorbid negative symptomatology.

\section{References}

1. Kiberstis P, Roberts L. It's not just the genes. Science 2002; 296: 685 .

2. Ben-Shlomo Y, Kuh D. A life course approach to chronic disease epidemiology: conceptual models, empirical challenges and interdisciplinary perspectives. Int J Epidemiol 2002; 31: 285-293.

3. Fanous AH, Kendler KS. Genetic heterogeneity, modifier genes, and quantitative phenotypes in psychiatric illness: searching for a framework. Mol Psychiatry 2005;10: 6-13.

4. Rodríguez-Solano JJ, Gonzalez-de-Chavez M. Premorbid personality disorders in schizophrenia. Schizophr Res 2000; 44: 137-44.

5. Keshavan MS, Duggal HS, Veeragandham G, McLaughlin NM, Montrose DM, et al. Schooler NE. Personality dimensions in first-episode psychoses. Am J Psychiatry $2005 ; 162: 102-109$.

6. Tandon R, DeQuardo JR, Taylor SF, McGrath M, Jibson $\mathrm{M}$, Eiser A, et al. Phasic and enduring negative symptoms in schizophrenia: biological markers and relationship to outcome. Schizophr Res 2000; 45: 191-201.

7. Miller DD, Flaum M, Arndt S, Fleming F, Andreasen NC. Effect of antipsychotic withdrawal on negative symptoms in schizophrenia. Neuropsychopharmacology 1994; 11: 11-20.

8. Flaum M, Andreasen N. The reliability of distinguishing primary versus secondary negative symptoms. Compr Psychiatry 1995; 36: 421-427.
9. Peralta V, Cuesta MJ, Martinez-Larrea A, Serrano JF. Differentiating primary from secondary negative symptoms in schizophrenia: a study of neuroleptic-naive patients before and after treatment. Am J Psychiatry 2000; 157: 1461-1466.

10. Carpenter WT, Heinrichs DW, Alphs LD. Treatment of negative symptoms. Schizophr Bull 1985; 11: 440-452.

11. Möller HJ, Müller H, Borison RL, Schooler NR, Chouinard G. A path-analytical approach to differentiate between direct and indirect drug effects on negative symptoms in schizophrenic patients: a re-evaluation of the North American risperidone study. Eur Arch Psychiatry Clin Neurosci 1995; 245: 45-49.

12. Möller HJ, Müller H. Statistical differentiation between direct and indirect effects of neuroleptics on negative symptoms. Eur Arch Psychiatry Clin Neurosci 1997; 247: $1-5$.

13. Kelley ME, van Kammen DP, Allen DN. Empirical validation of primary negative symptoms: indepedence from effects of medication and psychosis. Am J Psychiatry 1999; 156: 406-411.

14. McCreadie RG, Connolly MA, Williamson DJ, Athawes RWB, Tilak-Sihgh D. The Nithsdale Schizophrenia Surveys. XII. Neurodevelopmental schizophrenia: A search for clinical correlates and putative aetiological factors. Br J Psychiatry 1994; 165: 340-346.

15. Baum KM, Walker E, Childhood behavioral patterns of adult symptom dimensions in schizophrenia. Schizophrenia Res 1995; 16: 111-120.

16. Cannon TD, Mednick SA, Parnas J. Antecedents of predominantly negative and predominantly positive symptom schizophrenia in a high risk population. Arch Gen Psychiatry 1990; 47: 622-632.

17. Jorgensen A., Parnas J. The Copenhagen high-risk study: premorbid and clinical dimensions of maternal schizophrenia. J Nerv Ment Dis 1990; 178: 370-376.

18. Cuesta, MJ, Peralta V, Caro F. Premorbid personality in psychoses. Schizophr Bull 1999; 25: 801-811.

19. Peralta V, Cuesta MJ. Psychometric properties of the positive and negative syndrome scale (PANSS) in schizophrenia. Psychiatry Res 1994; 53: 31-40.

20. Spitzer RL, Williams JB, Gibbon M, First MB. The Structured Clinical Interview for DSM-III-R (SCID): I. history, rationale, and description. Arch Gen Psychiatry 1992; 49: 624-629.

21. American Psychiatric Association.: Diagnostic and Statistical Manual of Mental Disorders: DSM-IV. 4th ed. Washington, DC: American Psychiatric Association, 1994. 
22. Cuesta MJ, Gil P, Artamendi M, Serrano JF, Peralta V. Premorbid personality and psychopathological dimensions in first-episode psychosis. Schizophr Res 2002; 58: 273-280.

23. Marder SR, Davis JM, Chouinard G. The effects of risperidone on the five dimensions of schizophrenia derived by factor analysis: combined results of the North American trials. J Clin Psychiatry 1997; 58: 538-546.

24. Simpson GM, Angus JW. A rating scale for extrapyramidal side effects. Acta Psychiatr Scand Special 1970; 212: 11-19.

25. Tyrer P. Personality disorder. Diagnosis, management and course. London: Wright, 1988.

26. van Mastrigt S, Addington J. Assessment of premorbid function in first-episode schizophrenia: modifications to the Premorbid Adjustment Scale. J Psychiatry Neurosci 2002; 27: 92-101.
27. Yung AR, Phillips LJ, Yuen, HP, McGorry, PD. Risk factors for psychosis in an ultra high-risk group: psychopathology and clinical features. Schizophr Res 2004; 67: 131-142.

28. Faraone SV, Green AI, Seidman LJ, Tsuang MT. Schizotaxia: clinical implications and new directions for research. Schizophr Bull 2001; 27: 1-18.

29. Owens DG, Miller P, Lawrie SM, Johnstone EC. Pathogenesis of schizophrenia: a psychopathological perspective. Br J Psychiatry 2005; 186: 386-393.

Address for correspondence:

Manuel J. Cuesta

Psychiatric Unit

Virgen del Camino Hospital

C/ Irunlarrea sn

E-31008, Pamplona (SPAIN)

FAX +34948 429924

Email: mcuestaz@cfnavarra.es 